\title{
Detection of Cryptosporidium - Specific Coproantigen in Human Immunodeficiency Virus/Acquired Immunodeficiency Syndrome Patients by Using a Commercially Available Immunoenzymatic Assay
}

\author{
Claudio Vieira Silva, Marcelo Simão Ferreira*/+, \\ Maria do Rosário de Fátima Gonçalves-Pires, Julia Maria Costa-Cruz
}

Laboratório de Parasitologia, Instituto de Ciências Biomédicas *Disciplina de Moléstias Infecciosas e Parasitárias, Faculdade de Medicina, Universidade Federal de Uberlândia, Av. Pará 1720, 38400-902 Uberlândia, MG, Brasil

The aim of this study was to verify the occurrence of Cryptosporidium infection in 52 human immunodeficiency virus (HIV)/acquired immunodeficiency syndrome (AIDS) patients (group 1) and 38 clinically healthy individuals (group 2) by using enzyme immunoassay (EIA). All fecal samples collected were submitted to the Baermann, Lutz, and Ritchie methods, the Safranin/Methylene Blue, and Weber's chromotrope modified Trichrome staining techniques, and EIA. In group 1, parasitological staining techniques and EIA were both positive for Cryptosporidium $s p$. infection in 3/52 (5.8\%) samples and both negative in 45/52 (86.5\%) samples, while 4/52 (7.7\%) samples were positive in EIA and negative in parasitological staining techniques. Concerning group 2, all samples were negative by EIA and microscopy for Cryptosporidium infection. In conclusion, EIA may be an alternative method for detecting Cryptosporidium-specific coproantigen in HIV/AIDS patients.

Key words: Cryptosporidium sp. - coproantigen - acquired immunodeficiency syndrome (AIDS)

Cryptosporidium sp. is recognized as a major cause of waterborne diarrheal disease worldwide. The gastrointestinal disturbances are self-limiting in immunocompetent hosts, but chronic infections in immunocompromised subjects may become life-threaten-ing (Bialek et al. 2002). Among patients with cryptosporidiosis, the majority of immunocompetent individuals are initially symptomatic, with large number of oocysts present in their stools. However, as the acute infection resolves and the patient becomes asymptomatic, the number of oocysts decreases. Also, the number of oocysts passed by patients, including those with acquired immunodeficiency syndrome (AIDS) or infected by human immunodeficiency virus (HIV), varies from day to day and week to week. It has also been established that the infective dose of Cryptosporidium oocysts in humans can be relatively low (DuPont et al. 1995, Garcia et al. 2000).

Failure to diagnose cryptosporidiosis in the immunocompetent patient with diarrhea will rarely be of consequence because the disease is usually self-limiting. In contrast, the diagnosis of cryptosporidiosis is essential

Financial support: Capes and Fundação de Amparo à Pesquisa do Estado de Minas Gerais, Brasil

${ }^{+}$Corresponding author. Fax: +55-34-3236.3151. E-mail: mferreira@nanet.com.br

Received 12 June 2003

Accepted 30 October 2003 in the immunocompromised patient because it may interfere with therapeutical procedures, even though no therapy of cryptosporidiosis is currently known (Bialek et al. 2002).

Cryptosporidium oocysts are not detectable by routine iodine staining of feces. Modified acid-fast and Safranin/Methylene Blue staining techniques disclose intestinal coccidian (Baxby et al. 1984). However, these methods are laborious, require skilful experience, and have low sensitivity as compared to enzyme immunoassay (EIA) for detecting antigen, which has been introduced successfully for Cryptosporidium screening in feces (Garcia \& Shimizu 1997). The qualitative immunoenzymatic ProSpecT Cryptosporidium sp. Microplate Assay (Alexon, Inc., Biobrás) was developed in the United States to detect a Cryptosporidium specific antigen (CSA) in fresh and formalin or sodium acetate-acetic acid-formalin (SAF) preserved stool eluates, which can be read visually or spectrophotometrically. However, stool samples treated with polyvinyl alcohol (PVA) are not suitable for this use.

The aim of this study was to verify the occurrence of Cryptosporidium infection in HIV/AIDS patients by using EIA. It was conducted according to the ethical guidelines of the Brazilian Health Ministry concerning research on human beings (Ministério da Saúde 1996) after being approved by the Research Ethics Committee of Federal University of Uberlândia, state of Minas Gerais, Brazil.

The patients selected for the study were separated into following groups: group 1 consisted of 52 HIV/AIDS patients diagnosed by enzyme linked immunosorbent assay (ELISA) (Organon) and microparticle enzyme immunoassay (MEIA) (Axsym) methods anti-HIV I/II and 
hospitalized in the Infectious and Parasitic Diseases Ward of the teaching Clinic Hospital, Federal University of Uberlândia. Of them, 31 (59.6\%) were male, 21 (40.4\%) female; none of them were under highly antiretroviral therapy (HAART) by choice. Their ages ranged from 24 to 65 years $(\mathrm{yr})$ old $[\mathrm{mean}=36.6 \mathrm{yr}$; median $=36 \mathrm{yr}$; standard desviation $(\mathrm{SD})=9.3 \mathrm{yr}]$ and presented mean CD4 T cell count of 141 cells $/ \mathrm{mm}^{3}(\mathrm{SD}=204.6$; range: 3 923 cells $/ \mathrm{mm}^{3}$ ). CD4 T cell counts from group 1 were available by consulting medical records. Group 2 consisted of 38 clinically healthy individuals and served as control group. Of them, 18 (47.4\%) were male, $20(52.6 \%)$ female. Their ages ranged from 18 to 59 years old [mean $30.7 \mathrm{yr} ;$ median $=30 \mathrm{yr} ; \mathrm{SD}=11.8 \mathrm{yr}]$.

Both groups were submitted to coprological exams of three fecal samples collected on alternate days. Universal collectors, without preservatives, were used. The fresh fecal samples were first processed by the Baermann (1917) method. After that, the samples were preserved in $10 \%$ formalin and processed by the Lutz (1919) and Ritchie (1948) methods and the Safranin/Methylene Blue (Baxby et al. 1984) and Weber's chromotrope modified Trichrome (Weber et al. 1992) staining techniques. All samples were submitted to the five parasitological methods cited above. Two slides for each method were performed making a total of ten slides per sample, which were examined by two investigators. A total of 2700 slides were analyzed in light microscope (Nikon, Japan) at 100, 400, and 1000X.

After parasitological procedures, the remaining specimen of the three stool samples of each patient was homogenized and EIA was performed according to the manufacturer's instructions (ProSpecT Microplate Assay, Alexon Inc.). Briefly, microtiter plate wells (provided with the kit) were incubated with $0.1 \mathrm{ml}$ of stool sample (diluted $1: 1$ in diluent provided with the kit) for $1 \mathrm{~h}$ at room temperature. After shaking out the contents of the well and washing three times in the wash solution, $0.2 \mathrm{ml}$ of enzymatic conjugate was added and incubated for $30 \mathrm{~min}$ at room temperature. The strips were washed five times and substrate solution was added and incubated at room temperature for $10 \mathrm{~min}$. Stop solution was then added and the strips were read in a microtiter plate reader (Metrolab, Argentina) at $450 \mathrm{~nm}$. Results were interpreted according to the kit instructions as visual readings considering negative when the reaction is colorless, which indicates that none or undetectable level of CSA is present in the sample tested. The positive reaction develops yellow color of variable intensity and spectrophotometric readings establishing positive tests when optical density (OD) values were $\geq 0.05$ after the blank of the negative control is subtracted, indicating that the sample tested contains CSA. The result is considered negative when it presents OD values $<0.05$.

Fecal consistence was diarrheic in $24(46 \%)$ of the samples from group 1 and all the samples were nondiarrheic in group 2. Parasites were detected by parasitological methods in $12(23.1 \%)$ patients from group 1 and $10(26.3 \%)$ from group 2. The intestinal parasites found in group 1 were: Isospora belli (4/52), Cryptosporidium sp. (3/52), Strongyloides stercoralis (3/52), hookworm (1/52), and Taenia sp. (1/52). One case of coinfection was observed in group 1, involving $S$. stercoralis and $I$. belli. In relation to group 2 , the intestinal parasites and commensals verified were: hookworm (3/ 38), Entamoeba coli (3/38), Trichuris trichiura (1/38), Ascaris lumbricoides (1/38), Giardia lamblia (1/38), and Endolimax nana (1/38).

Concerning group 1, parasitological staining techniques and EIA were both positive for Cryptosporidium sp. infection in $3 / 52(5.8 \%)$ samples and both negative in 45/52 (86.5\%) samples, while 4/52 (7.7\%) samples were positive in EIA and negative in parasitological staining techniques. In group 2, all samples were negative by EIA and microscopy for Cryptosporidium infection.

Figure represents the detection of Cryptosporidium coproantigen by EIA in stool samples from groups 1 and 2. In group 1 the OD varied from 0.00 to 0.03 in the negative specimens and from 0.09 to 1.27 in the positive ones. All samples from group 2 were negative and the OD varied from 0.00 to 0.03 .

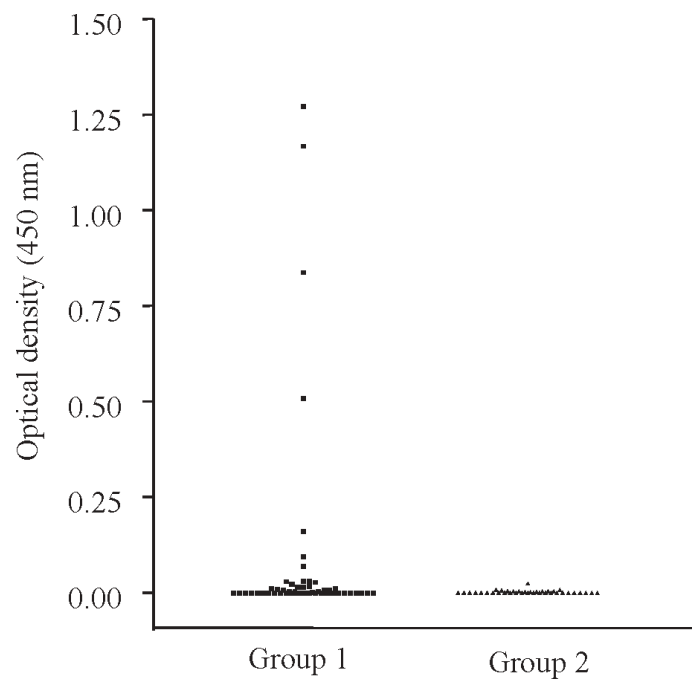

Detection of Cryptosporidium coproantigen by using the ProSpecT Cryptosporidium sp. Microplate Assay (spectrophotometric readings) in fecal samples from (ष) 52 HIV/AIDS patients (group 1) and (A) 38 individuals from control group (group 2). Cut off $=0.05$

The main signs and symptoms found in the Cryptosporidium infected patients from group 1 were chronic diarrhea $(6 / 7 ; 85.7 \%)$, weight loss $(5 / 7 ; 71.4 \%)$, vomiting $(3 / 7 ; 42.8 \%)$, nausea $(2 / 7 ; 28.6 \%)$, and anorexia $(2 / 7 ; 28.6 \%)$. One asymptomatic case was detected in both microscopy and EIA. Mean CD4 T cell count of patients infected with Cryptosporidium was 23.3 cells $/ \mathrm{mm}^{3}(\mathrm{SD}=13.5$; range: $10-50$ cells $/ \mathrm{mm}^{3}$ ). Four (57.1\%) of them died from AIDSrelated causes.

Diagnosis of cryptosporidiosis relies on recognition of the parasite by conventional light microscopy. This is laborious and insensitive, and may be prone to error if staff is inexperienced (Okhuysen 2001). High sensitivity, specificity, positive and negative predictive values have been reported for EIA (Kehl et al. 1995, Katanik et al. 
2001). In this study, EIA detected four positive cases of cryptosporidiosis that were not verified by microscopy. By consulting medical records, we could verify that these four patients were not under antiretroviral therapy, their CD4 T cell counts were $<50$ cells $/ \mathrm{mm}^{3}$ and that research for bacteria and viruses was negative. The three stool samples collected from these patients were persistently negative for any intestinal parasite. As these patients had aqueous diarrhea it was difficult to collect a large amount of the specimen. Thus, the samples collected were repeatedly diluted for testing in five parasitological methods advocated here, what could have interfered with the sensitivity of the parasitological assays.

The ProSpecT Cryptosporidium Microplate Assay uses monoclonal antibody directed against a specific Cryptosporidium antigen, what reduces the possibility of cross-reactivity. The literature does not report crossreactions of anti-CSA antibody with other intestinal parasites (Kehl et al. 1995, Katanik et al. 2001). This fact was confirmed in this study, since no cross-reactivity with the intestinal parasites detected in both groups was found.

Both assays, parasitological and EIA, are easy to perform. Formalin ethyl acetate concentration or sedimentation by centrifugation is recommended for the initial processing of the specimen prior to perform staining techniques. A predilution step is required in EIA. These pretesting procedures increase the complexity of these methods and the time required to perform the tests. The ease for interpretation of results varied considerably in each assay. Safranin/Methylene Blue stained smears were difficult to interpret, requiring frequent examination at 1000X magnification in oil immersion to identify the organisms. EIA can be read visually, eliminating the need for spectrophotometer. However, spectrophotometric readings are helpful in determining the results of borderline specimens (Kehl et al. 1995). The high agreement found between visual and spectrophotometric readings (100\%) eliminates the need of a microplate reader, allowing its use by laboratories that do not have the cited equipment or for epidemiological studies.

The persistence of low-grade and chronic infections may have important implications for immunocompromised hosts (Thompson \& Chalmers 2002). Thus, the detection of one asymptomatic case of cryptosporidiosis reinforces the importance of screening for Cryptosporidium sp. in HIV/AIDS patients even without presenting diarrhea.

Host immunity plays a major role in limiting the consequences of a primary infection and this is evident from the life-threatening nature of the disease in individuals with an impaired immune system (Thompson \& Chalmers 2002). In HIV-infected patients with CD4 T cell counts > 200 cells $/ \mathrm{mm}^{3}$, Cryptosporidium infection may resolve spontaneously; however, in the later stages of HIV disease (CD4 T cell count $<100$ cells $/ \mathrm{mm}^{3}$ ), chronic infection may lead to dehydration and malnutrition frequently resulting in death (Okhuysen 2001). All Cryptosporidium infected patients of this study presented CD4 T cell count $<100$ cells $/ \mathrm{mm}^{3}$, what may explain the chronic character of diarrhea, weigh loss and death observed in the majority of the patients.

ProSpecT enzyme immunoassay is simple to perform, requires minimal training, and can be used for singlespecimen or batch-testing approaches, thus providing diagnostic laboratories with an alternative method for detecting CSA in patients fecal samples from HIV/AIDS patients, specially those who present cryptosporidiosislike symptoms and the results of the fecal examination are repeatedly negative.

\section{REFERENCES}

Baermann G 1917. Eine Einfache Methode zur Auffindung von Ankylostomum (Nematoden) Larven in Erdproben, Mededeel. mit h. Geneesk, Lab Weltevreden Feestbundel, Batavia, p. 41-47.

Baxby D, Blundell N, Hart CA 1984. The development and performance of a simple, sensitive method for the detection of Cryptosporidium oocysts in faeces. J Hyg 93: 317-323.

Bialek R, Binder N, Dietz K, Joachim A, Knobloch J, Zelck UE 2002. Comparison of fluorescence, antigen and PCR assays to detect Cryptosporidium parvum in fecal specimens. Diagn Microbiol Infect Dis 43: 283-288.

DuPont HL, Chappell CL, Sterling CR, Okhuysen PC, Rose JB, Jakubowski W 1995. The infectivity of Cryptosporidium parvum in healthy volunteers. $N$ Engl $J$ Med 332: 855-859.

Garcia LS, Shimizu RY 1997. Evaluation of nine immunoassay kits (enzyme immunoassay and direct fluorescence) for detection of Giardia lamblia and Cryptosporidium parvum in human fecal specimens. J Clin Microbiol 35: 1526-1529.

Garcia LS, Shimizu RY, Bernard CN 2000. Detection of Giardia lamblia, Entamoeba histolytica/Entamoeba dispar, and Cryptosporidium parvum antigens in human fecal specimens using the triage parasite panel enzyme immunoassay. J Clin Microbiol 38: 3337-3340.

Katanik MT, Schneider SK, Rosenblatt JE, Hall GS, Procop GW 2001. Evaluation of ColorPAC Giardia/Cryptosporidium rapid assay and ProSpecT Giardia/Cryptosporidium microplate assay for detection of Giardia and Cryptosporidium in fecal specimens. J Clin Microbiol 39: 4523-4525.

Kehl KS, Cicirello H, Havens PL 1995. Comparison of four different methods for detection of Cryptosporidium species. J Clin Microbiol 33: 416-418.

Lutz AV 1919. Schistosoma mansoni e a schistosomose, segundo observações feitas no Brasil. Mem Inst Oswaldo Cruz. 11: 121-125.

Ministério da Saúde 1996. Resolução No 196/96 sobre pesquisa envolvendo seres humanos. Conselho Nacional de Saúde, Brasília.

Okhuysen PC 2001. Traveler's diarrhea due to intestinal protozoa. Clin Infect Dis 33: 110-114.

Ritchie LS 1948. An ether sedimentation technique for routine stool examination. Bull US Army Med Dept 8: 326-334.

Thompson RC, Chalmers RM 2002. Cryptosporidium: from molecules to disease. Trends Parasitol 18: 98-100.

Weber R, Bryan RT, Owen RL, Wilcox CM, Gorelkin L, Visvesvara GS 1992. Improved light-microscopical detection of microsporidia spores in stool and duodenal aspirates. $N$ Engl J Med 326: 161-166. 